\title{
STIFF PERSON SYNDROME: CASE REPORT.
}

Isabella Matias Ribeiro (UFRJ- Campus Macaé, Macaé, RJ, Brasil), Flávio Ribeiro Pereira (UFRJ- Campus Macaé, Macaé, RJ, Brasil), Jannine Farias Bellini Leite (UFRJ- Campus Macaé, Macaé, RJ, Brasil), Fernanda Marvila Fagundes Lamarca (UFRJ- Campus Macaé, Macaé, RJ, Brasil), Ingrid Carvalho Andrade (UFRJCampus Macaé, Macaé, RJ, Brasil), Adail Orrith Liborio Neto (UFRJ- Campus Macaé, Macaé, RJ, Brasil)

\section{BACKGROUND}

Stiff Person syndrome is a rare disease characterized by progressive increase of muscle tone, stiffness and painful spasms predominantly in the axial musculature. This clinical entity may be presented as a paraneoplastic syndrome and/or be correlated with other autoimmune diseases.

\section{CASE REPORT}

V.C.P.A, 54 years old, female, divorced, complaining of arthralgia in feet, paresthesia, muscle stiffness, difficulty in walking and frequent falls 1 year ago. She refers, initially, to have been evaluated by the neurologist who proposed the diagnosis of rheumatoid arthritis. It was prescribed Benzodiazepine and antidepressant, presenting partial improvement of the symptoms and returning to roam, even weakly. At the physical exam, she presented diffuse arthralgia, difficulty of roaming and reduction of strength in lower limbs. Laboratory tests were requested and demonstrated high levels of ESR (55) and CRP (6.8), rheumatoid factor 10,124 IU/mL, anti-Ro $237 \mathrm{U} / \mathrm{mL}$, non-reactive FAN and anti-acetylcholine antibody, without complement consumption, non-reactive viral serologies and normal thyroid hormones, hepatogram and renal function. Chest X-ray revealed sindesmophyte in the thoracic spine, with a dorsal traum in the middle lobe of the right lung. For diagnostic elucidation, an electromyography of the lower limb was requested, which revealed muscle spasms in all muscles, but more intense in the lumbosacral segment, without neuropathy, suggesting pathologies for continuous muscular rigidity. In view of the clinical investigation, the doctors concluded that it was Stiff Person syndrome. Clonazepam $1 \mathrm{mg} 8 / 8 \mathrm{~h}$ and Duloxetine $60 \mathrm{mg} /$ day were established as therapy. Tomography of the thorax, abdomen and pelvis were requested, which evidenced only hypodense nodular formation in hepatic segment VIII, measuring about $13 \mathrm{~mm}$, suggestive of hemangioma; there wasn't other alterations in the remaining parts. Endoscopy and colonoscopy were also without abnormalities. The mammography classified as BI-RADS 2, excluded neoplasia.

\section{CONCLUSION}

The diagnosis of Stiff Person syndrome is not simple, especially since it is a rare autoimmune entity, which can be associated with other autoimmune diseases, such as type 1 diabetes mellitus and hypothyroidism. This condition may also be associated with malignant tumor pathologies, however, in the majority of cases it's assumed as an isolated autoimmune etiology, as reported in this case. 